\title{
Continuous-Time Proportional Hazards Regression for Ecological Monitoring Data
}

\author{
Feng-Chang Lin [Postdoctoral Research Associate] and \\ Department of Biostatistics, University of North Carolina at Chapel Hill, Chapel Hill, NC 27599, \\ USA

\section{Jun Zhu [Professor]} \\ Department of Statistics and Department of Entomology, University of Wisconsin at Madison, \\ Madison, WI 53706, USA
}

\begin{abstract}
We consider a continuous-time proportional hazards model for the analysis of ecological monitoring data where subjects are monitored at discrete times and fixed sites across space. Since the exact time of event occurrence is not directly observed, we rely on dichotomous event indicators observed at monitoring times to make inference about the model parameters. We use autoregression on the response at neighboring sites from a previous time point to take into account spatial dependence. The interesting fact is utilized that the probability of observing an event at a monitoring time when the underlying hazards is proportional falls under the class of generalized linear models with binary responses and complementary log-log link functions. Thus, a maximum likelihood approach can be taken for inference and the computation can be carried out using standard statistical software packages. This approach has significant computational advantages over some of the existing methods that rely on Monte Carlo simulations. Simulation experiments are conducted and demonstrate that our method has sound finite-sample properties. A real dataset from an ecological study that monitored bark beetle colonization of red pines in Wisconsin is analyzed using the proposed models and inference. Supplementary materials that contain technical details are available online.
\end{abstract}

\section{Keywords}

Bark beetle; Binary data; Forest ecology; Generalized linear model; Maximum likelihood; Spatial autoregression

\section{INTRODUCTION}

Time-to-event data arise naturally in ecological monitoring programs involving occur-rences of events that mark the various life stages of organisms. Even though there is a large body of literature about statistical analysis of time-to-event data in a variety of disciplines particularly engineering and medicine, we observe that survival models are not as widely used for ecological and environmental studies. The purpose of this paper is to fill some of

(c) 2012 International Biometric Society

(flin@bios.unc.edu), (jzhu@stat.wisc.edu).. SUPPLEMENTARY MATERIALS

Appendix: The regularity conditions and a proof of Theorem 1 are given in a PDF. 
this void by providing a practical statistical method for the analysis of ecological monitoring data observed over time at fixed monitoring sites across space.

The motivating example is a study of forest declines that can have large effect on both timber production and successional trajectories of forest ecosystems. Gaining understanding of the mechanisms behind forest declines facilitates policy and management decisions and is of great importance. For instance, abiotic factors, such as soil characteristics and drought stress, can predispose trees to biotic mortality agents, such as insects and root pathogens (Klepzig, Raffa, and Smalley 1991; Erbilgin and Raffa 2002; Aukema et al. 2010). Of particular interest is a study of the impact of two bark beetle groups, turpentine beetles which generally do not kill a tree and Ips spp. which are capable of killing mature trees en masse, on the decline of red pines in a plantation in Wisconsin (Rasmussen et al. 2007; Zhu et al. 2008).

Two approaches were taken for the analysis of this bark beetle dataset in the past. Zhu et al. (2008) developed a set of spatial-temporal autologistic models, where the index of time is assumed to be discrete and coincides with the monitoring times. At the heart of this approach is Markov random field models, where the response variable at one spatial location and one point in time is modeled via a probability distribution conditional on neighboring locations and adjacent time points, giving rise to a valid joint distribution at all locations on the lattice and over time, under suitable regularity conditions. However, for a non-Gaussian distribution, the likelihood function of a Markov random field model contains an unknown normalizing constant which imposes serious computational difficulty (Zheng and Zhu 2008).

Rasmussen et al. (2007) proposed a spatial-temporal multivariate point process with an additive hazard function such that the index of time is continuous in the point process, even though monitoring times are discrete. In this alternative approach, the likelihood function has a closed form, provided that the baseline hazard is known a priori. Thus, the computation is faster than in an autologistic modeling approach. In related work, Nathoo (2010) developed a nonhomogeneous mixed Poisson process model for recurrent infection of trees by pine weevil, which is further linked with a spatial model representing the underlying height growth of the trees. Both Rasmussen et al. (2007) and Nathoo (2010) adopted a Bayesian hierarchical modeling framework for statistical inference and the computation can still be quite intensive. Conceptually, however, Rasmussen et al. (2007) contended that the continuous-time model has several advantages over the discrete-time models. In particular, the existence of an underlying continuous-time process is not always guaranteed for a discrete-time process, whereas this is a non-issue for a continuous-time model. Also, regression coefficients from continuous-time processes are comparable in scale even when monitoring times have different frequencies, which is not always the case for discrete-time processes on different monitoring time scales.

In this paper, we propose survival models in the continuous-time modeling framework and develop methodology that is practical in both implementation and interpretation for the analysis of the bark beetle data. The main innovations are as follows. First, we consider flexible forms for the hazard function, which naturally accounts for temporal correlation. In particular, we focus on proportional hazards and discuss an extension to additive hazards. We also relax the assumption in Rasmussen et al. (2007) that the baseline hazard function is known a priori, which can be quite restrictive in practice. Instead, we cast the problem in a modeling and inference framework where the baseline hazard function is specified in a flexible manner.

Second, we allow covariates to be time-heterogeneous in the regression, but only to a certain degree. This strategy enables us to strike a balance between model flexibility and 
computational feasibility. On the one hand, we allow covariates to vary from different monitoring periods. We also derive covariates from the response variables in the neighborhood from a previous time period, for the purpose of accounting for spatial dependence. That is, we include autoregressive terms in the model, rather than imposing an explicit spatial correlation structure on a random error term (see, e.g., Banerjee, Carlin, and Gelfand 2004; Diggle and Ribeiro 2007). Thus, our modeling approach is, in essence, to induce spatial dependence via autoregression (i.e., regression of the response at a given time point in time and site on those at previous time points and at neighboring sites). This approach is not uncommon. For example, autoregressive models are popular for time-series data (see, e.g., Box, Jenkins, and Reinsel 2008). In addition, our model is dynamic rather than static, in the sense the autoregression is on previous times but not current time point. This is a main idea behind some of the spatial dynamic models for spatial-temporal data (see, e.g., Wikle and Hooten 2006). In addition, we allow regression coefficients of these covariates to be time-heterogeneous, relaxing the time-homogeneous coefficient assumptions in both Rasmussen et al. (2007) and Zhu et al. (2008). On the other hand, we let covariates be time invariant within the same monitoring period. We believe that this is a relatively small price to pay for a substantial gain in computational efficiency, as we will demonstrate in Sections 2 and 3.

Third, we cast statistical inference in the maximum likelihood framework. Via an interesting connection between our proportional hazards models and generalized linear models, our method can be implemented using any existing statistical software package that features generalized linear models. As a result, the proposed methods here have substantial computational advantage over those in Rasmussen et al. (2007) and Zhu et al. (2008). In particular, we use the idea that a binary regression with a complementary log-log link function is a grouped-time version of the continuous-time proportional hazards model (McCullagh 1980). Although this idea has been applied in various disciplines (see, e.g., Fahrmeir and Tutz 2001), to the best of our knowledge, its application to the analysis of ecological monitoring data appears to be limited.

The remainder of the paper is organized as follows. In Section 2, we describe a continuoustime proportional hazards regression model with time-heterogeneous baseline hazards and time-heterogeneous regression coefficients. We then consider reduced models that have a periodic baseline hazard function and/or time-homogeneous regression coefficients. In Section 3, we propose stratified maximum likelihood for the estimation of model parameters. A simulation study that demonstrates our proposed method is described in Section 4, followed by an analysis of the bark beetle data in Section 5 . We describe possible extensions in Section 6.

\section{CONTINUOUS-TIME MODEL}

\subsection{A Full Model}

Let $t>0$ denote continuous time and $a_{k}$ denote the $k$ th monitoring time, where $k=0,1, \ldots$, $K$ and $a_{0}=0<a_{1}<\cdots<a_{K}<\infty$. Let $i$ index the subject at the $i$ th site in the study, where $i$ $=1, \ldots, n$ and $n$ is the total of number of sites. At the $k$ th monitoring time, it is known whether an event has occurred to an at-risk subject within the monitoring period $\left(a_{k-1}, a_{k}\right]$, for $k=1, \ldots, K$. Since the exact time of event is not known, the observations are interval censored. We use a dichotomous response variable to denote an observation as

$\delta_{k, i}=\mathscr{I}$ the subject at the $i$ th site is observed with an event at the $k$ th monitoring time $\left.a_{k}\right\}$, 
where $\mathscr{I}\{\cdot\}$ denotes an indicator function. We use another dichotomous variable to denote whether a subject is at risk or not for a given monitoring period $\left(a_{k-1}, a_{k}\right]$ as

$Y_{k, i}=\mathscr{I}$ the subject at the $i$ th site is at risk in the $k$ th monitoring period\} .

In addition, we let $\boldsymbol{Z}_{k, i}$ denote a $p$-dimensional vector of covariates for the subject at the $i$ th site during the $k$ th monitoring period. Thus, the data consist of the triplets $\left(Y_{k, i}, Z_{k, i}, \delta_{k, i}\right)$, for $i=1, \ldots, n$ and $k=1, \ldots, K$.

To model the probability of an event occurring to the subject at the $i$ th site in the $k$ th monitoring period $\left(a_{k-1}, a_{k}\right]$, we use a proportional hazards function

$$
\lambda_{k, i}(t)=Y_{k, i} \lambda_{k, 0}(t) \exp \left(\beta_{k}^{\prime} \boldsymbol{Z}_{k, i}\right),
$$

where $\lambda_{k, 0}$ is a baseline hazard function of time $t$ and $\beta_{k}$ is a $p$-dimensional vector of regression coefficients, both for the $k$ th monitoring period with $k=1, \ldots, K$ (Cox 1972).

Model (2.1) is flexible in several aspects. The baseline hazard function $\lambda_{k, 0}$ is not restricted to any specific form and can vary for different monitoring periods, yet maximum likelihood estimation of the regression coefficients $\beta_{k}$ is still attainable. This is an improvement over previous work where the baseline hazard needs to be pre-specified using external data. Furthermore, the regression coefficients $\beta_{k}$ can vary over monitoring periods, adding another layer of flexibility to the model. We also allow covariates $\boldsymbol{Z}_{k, i}$ to vary among different monitoring periods, although they are assumed to be constant within a given monitoring period. This latter assumption is made for gaining substantial computational efficiency. From a practical perspective, however, this assumption is not all that unrealistic, as fully continuous-time covariates are often not available in ecological monitoring programs. Even if they are available, an average can be taken over the course of time within a monitoring period, which is not uncommon in monitoring data analysis.

\subsection{Reduced Models}

We consider two possible ways of reducing model (2.1) to more parsimonious models, which we will refer to as reduced models. First, we consider a baseline hazard function $\lambda_{0}(\cdot)$ $\equiv \lambda_{k, 0}(\cdot)$ that is invariant over different monitoring periods. A periodic baseline hazard function is a possibility when $\lambda_{k, 0}$ in (2.1) is renewed right after each monitoring time and has the same functional form for different monitoring periods. That is, consider a hazard function, in the form of

$$
\lambda_{k, i}(t)=Y_{k, i} \lambda_{0}\left(t-a_{k-1}\right) \exp \left(\beta_{k}^{\prime} \boldsymbol{Z}_{k, i}\right),
$$

for $a_{k-1} \leq t<a_{k}$, to have a periodic baseline hazard $\lambda_{0}(\cdot)$. It follows that $a_{1}=\cdots=a_{K} \equiv a$ in (3.1), under model (2.2) and provided that the length of each monitoring period is the same. Model (2.2) is a reduced model from (2.1), since the baseline hazard has only one parameter $a$.

Next, we consider regression coefficients $\beta \equiv \beta_{k}$ that are homogeneous over time. The hazard function in model (2.1) becomes

$$
\lambda_{k, i}(t)=Y_{k, i} \lambda_{k, 0}(t) \exp \left(\beta^{\prime} \boldsymbol{Z}_{k, i}\right) .
$$

Combining (2.2) and (2.3), we obtain the most parsimonious model 


$$
\lambda_{k, i}(t)=Y_{k, i} \lambda_{0}\left(t-a_{k-1}\right) \exp \left(\beta^{\prime} \boldsymbol{Z}_{k, i}\right) .
$$

In addition, we may consider hybrid cases where, for example, the coefficient for one covariate is time-homogeneous, but that for another is time-heterogeneous.

\section{STATISTICAL INFERENCE VIA MAXIMUM LIKELIHOOD}

\subsection{Likelihood Function}

When the subject at the $i$ th site is at risk in the $k$ th monitoring period (i.e., $Y_{k, i}=1$ ), the probability of an event (i.e., $\delta_{k, i}=1$ ) at $a_{k}$ under model (2.1) is

$$
\theta_{k, i}=1-\exp \left\{-\int_{a_{k-1}}^{a_{k}} \lambda_{k, i}(t) \mathrm{d} t\right\}=1-\exp \left\{-\alpha_{k} \exp \left(\beta_{k}^{\prime} \boldsymbol{Z}_{k, i}\right)\right\}
$$

where $\alpha_{k}=\int_{a_{k-1}}^{a_{k}} \lambda_{k, 0}(t) \mathrm{d} t, k=1, \ldots, K$. The second equality in (3.1) does not hold when $\boldsymbol{Z}_{k, i}$ varies over time between $a_{k-1}$ and $a_{k}$. Estimation would be more challenging if the covariates are fully time-dependent in this case, since the probability term $\theta_{k, i}$ in (3.1) may not have a closed form. Thus, we allow $\boldsymbol{Z}_{k, i}$ to differ in different monitoring periods $k$, but not to vary by time $t$ within the same monitoring period.

Let $\eta=\left(\alpha^{\prime}, \beta\right)^{\prime}$ with $a=\left(a_{1}, \ldots, a_{K}\right)^{\prime}$ and $\beta=\left(\beta_{1}^{\prime}, \ldots, \beta_{\mathrm{K}}^{\prime}\right)^{\prime}$ denote the vector of all the model parameters. Under a probability model $\theta_{k, i}$ for a dichotomous response $\delta_{k, i}$ at each monitoring time $a_{k}$, we adopt maximum likelihood for the statistical inference of $\eta$. The loglikelihood function is

$$
\ell(\eta)=\sum_{i=1}^{n} \sum_{k=1}^{K} Y_{k, i}\left\{\delta_{k, i} \log \theta_{k, i}+\left(1-\delta_{k, i}\right) \log \left(1-\theta_{k, i}\right)\right\},
$$

where the responses are assumed to be independent, conditional on the whole history of events and covariates. That is, the correlation among responses is assumed to be adequately captured by past observable history (or, in counting process terminology, filtration). This assumption is not unreasonable for ecological monitoring programs where responses are correlated either temporally (within a subject) and/or spatially (between subjects). A counting process approach defined by an intensity function such as (2.1) is well-suited for modeling temporal correlation, provided that the assumption of conditional independent increment is satisfied. As for spatial correlation, we rely on incorporating useful spatial information into the vector of covariates $\boldsymbol{Z}_{k, i}$ and assume that the covariates can capture the spatial correlation between subjects reasonably well.

\subsection{Maximum Likelihood Estimation}

Following the log-likelihood function $\ell(\eta)$ in (3.2), the score function for $\eta$, from differentiating $\ell(\eta)$, is

$$
\boldsymbol{S}(\eta)=\partial \ell(\eta) / \partial \eta=\sum_{\mathbf{i}=\mathbf{1} \mathbf{k}=\mathbf{1}}^{\mathbf{n}} \sum_{\mathbf{k}, \mathbf{i}} \boldsymbol{D}_{\mathbf{k}, \mathbf{i}} \mathbf{v}_{\mathbf{k}, \mathbf{i}}^{-1}\left(\delta_{\mathbf{k}, \mathbf{i}}-\theta_{\mathbf{k}, \mathbf{i}}\right),
$$

where $\boldsymbol{D}_{k, i}=\partial \theta_{k, i} / \partial \eta$ and $v_{k, i}=\theta_{k, i}\left(1-\theta_{k, i}\right)$. An information matrix, from the negative second-order derivative of $\ell(\eta)$, is given by 


$$
\boldsymbol{I}(\eta)=\sum_{i=1}^{n} \sum_{k=1}^{K} Y_{k, i} v_{k, i}^{-1} \boldsymbol{D}_{k, i} \boldsymbol{D}_{k, i}^{\prime}
$$

since $\mathrm{E}\left(\delta_{k, i} \mid \boldsymbol{Z}_{k, i}\right)=\theta_{k, i}$. The score function (3.3) and the information matrix (3.4) are not restricted to any specific form of $\theta_{k, i}$, but rather hold in general for dichotomous responses.

Under our model (3.1) for $\theta_{k, i}$, we have

$$
\boldsymbol{D}_{k, i}=\left(\begin{array}{c}
\left(\partial \theta_{k, i} / \partial \alpha_{k}\right) \mathbf{1}_{k \times 1} \\
\left(\partial \theta_{k, i} / \partial \beta_{k}\right) \mathbf{1}_{k \times p}
\end{array}\right)
$$

where $\partial \theta_{k, i} / \partial \alpha_{k}=\left(1-\theta_{k, i}\right) \exp \left(\beta_{k}^{\prime} \boldsymbol{Z}_{k, i}\right), \partial \theta_{k, i} / \partial \beta_{k}=\left(1-\theta_{k, i}\right) \alpha_{k} \exp \left(\beta_{k}^{\prime} \boldsymbol{Z}_{k, i}\right) \boldsymbol{Z}_{k, i}$, and $\mathbf{1}_{k \times p}$ is a $(K \times p)$-column vector with a $p$-column vector $\mathbf{1}_{1 \times p}$ in the $k$ th block and 0 everywhere else. Thus, $\boldsymbol{S}(\eta)=\left(\boldsymbol{S}_{\alpha}(\eta)^{\prime}, \boldsymbol{S}_{\beta}(\eta)^{\prime}\right)^{\prime}$ is the full score function, where $\boldsymbol{S}_{\alpha}(\eta)=$ $\left(S_{a 1}(\eta), \ldots, S_{a_{K}}(\eta)\right)^{\prime}$ with

$$
S_{\alpha_{k}}(\eta)=\partial \ell(\eta) / \partial \alpha_{k}=\sum_{i=1}^{n} Y_{k, i} \theta_{k, i}^{-1} \exp \left(\beta_{k}^{\prime} \boldsymbol{Z}_{k, i}\right)\left(\delta_{k, i}-\theta_{k, i}\right),
$$

and $S_{\beta}(\eta)=\left(S_{\beta_{1}}(\eta)^{\prime}, \ldots, S_{\beta_{K}}(\eta)^{\prime}\right)^{\prime}$ with

$$
\boldsymbol{S}_{\beta_{k}}(\eta)=\partial \ell(\eta) / \partial \beta_{k}=\sum_{i=1}^{n} Y_{k, i} \theta_{k, i}^{-1} \alpha_{k} \exp \left(\beta_{k}^{\prime} \boldsymbol{Z}_{k, i}\right) \boldsymbol{Z}_{k, i}\left(\delta_{k, i}-\theta_{k, i}\right),
$$

for $1 \leq k \leq K$. Furthermore, the information matrix $\boldsymbol{I}(\eta)$ is obtained via (3.4) evaluated at $\boldsymbol{D}_{k, i}$ in (3.5).

Define the maximum likelihood estimate as $\widehat{\eta}=\arg \max _{\eta} \ell(\eta)$. With a fixed $K$, we can show that $\hat{\eta}$ is consistent and $n^{1 / 2}(\widehat{\eta}-\eta)$ is asymptotically normal with mean $\mathbf{0}$ and variance $\boldsymbol{i}(\eta)^{-1}$, where $\boldsymbol{i}(\eta)=\lim _{n \rightarrow \infty} n^{-1} \boldsymbol{I}(\eta)$. Furthermore, $\boldsymbol{i}(\eta)$ achieves the Cramer-Rao lower bound and thus $\widehat{\eta}$ is efficient. We summarize its asymptotic properties in the following theorem. A proof is provided in the appendix as supplementary materials online.

Theorem 1. Under the regularity conditions in Appendix $A, \widehat{\eta}$ is a consistent estimator of $\eta$, and $n^{1 / 2}(\widehat{\eta}-\eta)$ converges in distribution to a normal variable with mean zero and a covariance matrix that can be consistently estimated by $n \boldsymbol{I}(\widehat{\eta})^{-1}$.

Our approach above has substantial computational advantages, since $\theta_{k, i}$ in (3.1) can be transformed to

$$
\log \left\{-\log \left(1-\theta_{k, i}\right)\right\}=\log \alpha_{k}+\beta_{k}^{\prime} \boldsymbol{Z}_{k, i}
$$

via a complementary log-log link function, as in a generalized linear model with binomial response (McCullagh and Nelder 1989; Fahrmeir and Tutz 2001). Moreover, it can be shown that $\ell(\eta)$ is a stratified log-likelihood function with the monitoring periods serving as strata. Therefore, using function $\operatorname{glm}()$ in $\mathrm{R}$ with binomial family and cloglog link function 
would provide the desired parameter estimates. The stratification of the likelihood is achieved by adding indicator variables in the model fitting.

\subsection{Model Assessment and Comparison}

Models (2.1)-(2.4) are candidate models of interest. In order to determine which one is closer to the true model based on data, we may employ information criteria, such as Akaike's information criterion (AIC), since we take a maximum likelihood approach. Here the AIC is defined as $-2 \ell(\eta)+2 \#(\eta)$, where $\eta$ is the vector of parameters in a candidate model and $\#(\eta)$ is the number of parameters as the length of the vector. Models (2.1)-(2.4) have $\#(\eta)=K(p+1), K p+1, K+p$, and $p+1$, respectively. Furthermore, for a generalized linear model, goodness-of-fit tests are possible for evaluating the quality of the fit, whereas model diagnostics using residuals can be performed to evaluate the model assumptions (McCullagh and Nelder 1989).

\section{SIMULATION}

In this section, we conduct simulation experiments under different numbers of monitoring periods and sample sizes, in order to demonstrate the finite-sample properties of the maximum likelihood estimation. We consider the following proportional hazards function:

$$
\lambda_{k, i}(t)=\lambda_{k, 0}(t) \exp \left(\beta_{1} Z_{k, i}^{(1)}+\beta_{2} Z_{k, i}^{(2)}\right),
$$

where $Z_{k, i}^{(1)}$ are randomly generated by a Poisson distribution with mean 0.8 . To account for spatial dependence, we adopt autoregression. Define the $k$ th-order neighborhood of a given site as those sites that are the $k$ th nearest neighbors. For example, the first-order neighborhood on a square lattice has the four nearest neighbors in the north, south, west, and east, and the second-order neighborhood has the four second-nearest neighbors in the northwest, southwest, northeast, and southeast, etc. Let $Z_{k, i}^{(2)}=\sum_{j \in N_{i}} \delta_{k-1, j}$ be the total number of events in the neighborhood $N_{i}$ at a previous monitoring time. Here we consider $N_{i}$ as the set of the neighbors from the first to the fifth order. We assume $a_{0}=0$ at the onset of the monitoring and the subsequent monitoring times are $a_{k}=k$ for $k=1, \ldots, K$. The parameters are set to $\left(\beta_{1}, \beta_{2}\right)^{\prime}=(0.03,0.03)^{\prime}$, which are time-homogeneous, and $\alpha_{k}=\int_{a_{k-1}}^{a_{k}} \lambda_{k, 0}(t) \mathrm{d} t=0.1 \times k$.

Table 1 shows the simulation results based on combinations of the number of monitoring times $K$ and the number of sites $n=m^{2}$ on an $m \times m$ lattice, where $K=2,4,8$ and $m=20$, 30, 40. Each combination of $K$ and $n$ is replicated 1,000 times. We report the empirical relative bias (RB), empirical variance (EV), and average of variance estimates (AVE), which are defined as the average of replicated estimates minus the true value of $\beta$, the sample variance of replicated estimates, and the average of the variance estimates computed via the estimated information matrix, respectively. The empirical coverage probability $(\mathrm{CP})$ is also reported to see how close the confidence interval for $\beta$ is to the 0.95 nominal level.

Clearly, by the simulation results in Table 1, the maximum likelihood estimation performs well under each combination of $K$ and $m$. The bias and variance decrease when the sample size increases, which suggests that the estimator is asymptotically consistent. Moreover, the empirical variance estimation by the inverse of information matrix shown in the AVE column is close to the empirical variance of the estimates in the EV column. This suggests that the variance is well-approximated by inverting the information matrix evaluated at the maximum likelihood estimates. Finally, the closeness of the empirical coverage probability 
to the 0.95 nominal level shows that a Wald-type hypothesis test for the parameters can reach a desired level.

\section{BARK BEETLE DATA EXAMPLE}

We return to the data example where two bark beetle groups were studied in a red pine plantation of Wisconsin. In this study, a dataset of annual surveys of all trees in the plantation was available. Each year, each of the 2,715 trees was examined for tree condition (alive/dead), presence/absence of Ips spp., and number of pitch tubes each of which signifies colonization by a turpentine beetle. Among several important ecological questions, one of the most pressing was the degree of association between turpentine beetle and Ips spp., in terms of the likelihood that a tree colonized by turpentine beetles will be subsequently colonized by Ips spp. It was hypothesized that turpentine beetles act as a predisposer to colonization by Ips spp., a significant biotic factor that is strongly associated with the mortality of red pine trees.

We fit models (2.1), (2.3), and (2.4) to this dataset. Colonization of a tree by Ips spp. is considered to be an event. We let $a_{0}=0$ denote the beginning of the first year and $a_{k}$ denote the $k$ th monitoring time which occurs in the $k$ th year, where $k=1, \ldots, K=5$. The subjects are the red pine trees in the study area and there were a total of 2,715 sites of red pines in the beginning of the study. For a red pine that was colonized by Ips spp. during a previous monitoring period, it would no longer be at risk in the current monitoring period, because Ips spp. are known to kill a tree shortly after colonization and do not colonize the same tree twice. Thus, for such a red pine tree, the hazard is set to 0 . For a red pine that is at risk in the current monitoring period, however, there is a probability for the tree to be colonized by Ips spp. and this probability may be influenced by various factors.

For the purpose of our study, we consider the number of turpentine beetles that colonized the same tree as a possible covariate $\left(Z_{k, i}^{(1)}\right)$. Thus, whether the regression coefficients $\beta_{k}^{(1)}$ are significantly different from 0 or not would put the hypothesis that colonization by turpentine beetles predisposes that by Ips spp. to test. In addition, to account for spatial dependence, we count the number of trees that were colonized by Ips spp. in the neighborhood from a previous year and use this count as another possible covariate $\left(Z_{k, i}^{(2)}\right)$. As we discussed in Section 1, this approach of accounting for spatial dependence is different from the approach taken in Zhu et al. (2008). In Markov random field modeling, spatial dependence is modeled via autoregression within the same monitoring period and thus is static. The approach adopted here is more common in dynamic models, where spatial dependence is still via autoregression but on a previous time point.

Specifically, we first consider the full continuous-time proportional hazards model (2.1)

$$
\lambda_{k, i}(t)=Y_{k, i} \lambda_{k, 0}(t) \exp \left(\beta_{k 1} Z_{k, i}^{(1)}+\beta_{k 2} Z_{k, i}^{(2)}\right),
$$

where $Z_{k, i}^{(1)}$ is the number of turpentine beetles colonizing the tree at the $i$ th site by the $k$ th year, $Z_{k, i}^{(2)}$ is the number of trees with Ips colonization in a neighborhood up to the fifth order by the previous year $(k-1)$, and $\beta_{k}=\left(\beta_{k 1}, \beta_{k 2}\right)^{\prime}$ is a vector of time-heterogeneous regression coefficients. The notation for the two reduced models (2.2) and (2.4) is analogous. In addition, we consider a hybrid case where the coefficient $\beta_{k 1} \equiv \beta_{1}$ is time-homogeneous, but $\beta_{k 2}$ is time-heterogeneous. 
Table 2 shows the data analysis results. As discussed before, AIC is used to evaluate these competing models and select models with smaller AIC values. From the result, the model with heterogeneous baseline hazards (different $\alpha_{k}$ ), homogeneous coefficient $\beta_{1}$ for the number of turpentine beetles $\left(Z_{k, i}^{(1)}\right)$, and heterogeneous coefficients $\beta_{k 2}$ for the number of trees with Ips spp. in the neighborhood from a previous year $\left(Z_{k, i}^{(2)}\right)$, is the best model with the smallest AIC value. The results are similar when using AICC and are omitted here. This suggests that the effect of the Ips spp. colonization of the neighbors in a previous year differed over years, but that of the colonization of turpentine beetles was similar over time. Moreover, based on a Wald-type test for the regression coefficients, both covariates are significant. This provides evidence that the colonization of turpentine beetles predisposed colonization by Ips spp., as was initially hypothesized. There is also a positive feedback effect, as a higher chance of Ips spp. colonization of any given red pine in a given year was associated with a larger number of trees in the neighborhood with Ips spp. colonization from a previous year. The findings from the data analysis are qualitatively the same as before. Finally, the baseline hazards differed for different years and did not appear to be periodic.

Our method here is substantially easier to implement and faster to compute than the previous method based on spatial-temporal autoregressive model (Zhu et al. 2008), as well as that based on continuous-time model (Rasmussen et al. 2007). In addition, because of the greater computational ease, we are able to explore models with homogeneous or heterogeneous coefficients, as well as homogeneous or heterogeneous baseline hazards, which neither Rasmussen et al. (2007) nor Zhu et al. (2008) pursued.

For model diagnostics, we examine the deviance residuals and find that there is no obvious remainder spatial dependence left in the residual after the model fit based on empirical variograms (Cressie 1993). A similar approach is taken to examine temporal dependence, but the conclusion that there is no evidence of temporal dependence may be tenuous due to the small number of monitoring times. Furthermore, we utilize a quasi-likelihood approach to assess possible overdispersion by adding a scale parameter to the variance term $v_{k, i}=$ $\phi \theta_{k, i}\left(1-\theta_{k, i}\right)$ in (3.3) (Fahrmeir and Tutz 2001). The estimate of $\phi$ is 0.7 and there is no indication of overdispersion. Finally, we perform a goodness-of-fit (GOF) $\chi^{2}$ test using the residual deviance and obtain a $p$-value less than 0.001 . This indicates that there is room for improvement of the model fit. However, for binary outcomes that are rare events as in this dataset, the validity of such a GOF test is unclear.

\section{DISCUSSION}

In this paper, we have focused on a continuous-time proportional hazards model for the analysis of ecological monitoring data where subjects are monitored at discrete times and fixed sites across space. We have utilized the fact that the probability of observing an event at a monitoring time when the underlying hazards is proportional falls under the class of generalized linear models with binary responses and complementary log-log link functions. Thus, we have used a maximum likelihood approach for inference, which can be carried out via standard statistical software packages. This approach has significant computational advantages over some of the existing methods that rely on Monte Carlo simulations. The simulation experiments show that our method has sound finite-sample properties. We have applied this method to analyze an ecological monitoring dataset. Even though the scientific question in the bark beetle study has compelled us to formulate it as a regression problem, it is worth noting that other modeling approaches may be viable, such as a bivariate model that treats Ips. spp. and turpentine beetles as two random processes jointly (Nathoo 2010). 
We have stressed that model (2.1) has a continuous-time index and thus, in general, the hazard function $\lambda_{k, i}(t)$ is not the same as $\lambda_{k, i}\left(t^{\prime}\right)$ for $t \neq t^{\prime}$ even when $t$ and $t^{\prime}$ are within the same monitoring period $\left(a_{k-1}, a_{k}\right]$. For the purpose of estimating the regression coefficients $\beta_{k}$, we have computed a probability $\theta_{k, i}$, which integrates over the continuous-time interval $\left(a_{k-1}, a_{k}\right]$. This probability is time invariant within the same monitoring period for a given site, because of the assumptions made on the covariate vector $\boldsymbol{Z}_{k, i}$. In other words, the hazard cannot be fully evaluated on a continuous-time scale. However, we refrain from abandoning the continuous-time index $t$, as the definition of the hazard or probability is unclear and may not be valid if a discrete-time index $k$ is to replace $t$. This is a subtle point worth further investigation. Along this line, it would be interesting to extend our methodology to a fully continuous-time index while keeping the computational cost under control.

Further extensions or generalizations of our method are possible. For example, it is straightforward to extend to a continuous-time additive hazards model, in which case we observe that the probability $\theta_{k, i}=\mathrm{E}\left(\delta_{k, i} \mid \boldsymbol{Z}_{k, i}\right)$ can be expressed as

$$
-\log \left(1-\theta_{k, i}\right)=\alpha_{k}+\beta_{k}^{\prime} \boldsymbol{Z}_{k, i}, \quad(6.1)
$$

which is a generalized linear model with a complementary log link function (Piegorsch 1992; Fahrmeir and Tutz 2001). Moreover, while we have incorporated spatial correlation via observable spatial covariates, other approaches to account for spatial variation are of interest. Finally, in some practical situations, the monitoring periods are not equally spaced over time. Our methodology, in theory, can be extended to address this issue, but we anticipate the computational burden to increase greatly, due to an increasing number of intercept terms $a_{k}$ in (3.3). We leave this and other possible extensions for future research.

\section{Supplementary Material}

Refer to Web version on PubMed Central for supplementary material.

\section{Acknowledgments}

The authors are grateful to the editor, Professor Carl Schwarz, an associate editor, and two anonymous referees for their constructive comments, which helped improve the paper. We also thank Professor Ken Raffa for providing the bark beetle data used for illustration of our methodology. Funding has been provided for this research from a USDA Cooperative State Research, Education and Extension Service (CSREES) Hatch project and an NSF project.

\section{REFERENCES}

Aukema BH, Zhu J, Møller J, Rasmussen J, Raffa KF. Predisposition to Bark Beetle Attack by Root Herbivores and Associated Pathogens: Roles in Forest Decline, Gap Formation, and Persistence of Endemic Bark Beetle Populations. Forest Ecology and Management. 2010; 259:374-382.

Banerjee, S.; Carlin, BP.; Gelfand, AE. Hierarchical Modeling and Analysis for Spatial Data. Chapman and Hall/CRC; Boca Raton: 2004.

Box, G.; Jenkins, GM.; Reinsel, G. Time Series Analysis: Forecasting and Control. 4th ed.. Wiley; New York: 2008.

Cox DR. "Regression Models and Life-Tables" (with discussion). Journal of the Royal Statistical Society, Series B. 1972; 34:187-220.

Cressie, N. Statistics for Spatial Data. Revised ed.. Wiley; New York: 1993.

Diggle, PJ.; Ribeiro, PJ. Model-Based Geostatistics. Springer; New York: 2007.

Erbilgin N, Raffa KF. Association of Declining Red Pine Stands With Reduced Populations of Bark Beetle Predators, Seasonal Increases in Root Colonizing Insects, and Incidence of Root Pathogens. Forest Ecology and Management. 2002; 164:221-236. 
Fahrmeir, L.; Tutz, G. Multivariate Statistical Modelling Based on Generalised Linear Models. 2nd ed.. Springer; New York: 2001.

Klepzig KD, Raffa KF, Smalley EB. Association of Insect-Fungal Complexes With Red Pine Decline in Wisconsin. Forest Science. 1991; 41:1119-1139.

McCullagh P. "Regression Models for Ordinal Data" (with discussion). Journal of the Royal Statistical Society. Series B. 1980; 42:109-142.

McCullagh, P.; Nelder, J. Generalized Linear Models. 2nd ed.. Chapman and Hall/CRC; Boca Raton: 1989.

Nathoo FS. Joint Spatial Modeling of Recurrent Infection and Growth With Processes Under Intermittent Observation. Biometrics. 2010; 66:336-346. [PubMed: 19673862]

Piegorsch WW. Complementary Log Regression for Generalized Linear Models. The American Statistician. 1992; 46:94-99.

Rasmussen JG, Møller J, Aukema BH, Raffa KF, Zhu J. Bayesian Inference for Multivariate Point Processes Observed at Sparsely Distributed Times. Journal of the Royal Statistical Society. Series B. 2007; 69:701-713.

Wikle, CK.; Hooten, MB. Hierarchical Bayesian Spatio-Temporal Models for Population Spread. In: Clark, JS.; Gelfand, A., editors. Applications of Computational Statistics in the Environmental Sciences: Hierarchical Bayes and MCMC Methods. Oxford University Press; Oxford: 2006.

Zheng Y, Zhu J. Markov Chain Monte Carlo for a Spatial-Temporal Autologistic Regression Model. Journal of Computational and Graphical Statistics. 2008; 17:123-137.

Zhu J, Rasmussen J, Møller J, Aukema BH, Raffa KF. Spatial-Temporal Modeling of Forest Gaps Generated by Colonization From Below- and Above-Ground Bark Beetle Species. Journal of the American Statistical Association. 2008; 103:162-177. 


\section{Table 1}

Summary statistics of empirical relative bias (RB), empirical variance (EV), average of variance estimates (AVE), and empirical coverage probability (CP) for a 0.95 nominal level in the simulation study with varying number of monitoring times $(K)$ and lattice size $(m \times m)$.

\begin{tabular}{|c|c|c|c|c|c|c|c|c|c|}
\hline \multirow[b]{2}{*}{$\boldsymbol{K}$} & \multirow[b]{2}{*}{$m$} & \multicolumn{4}{|c|}{$\hat{\beta}_{1}$} & \multicolumn{4}{|c|}{$\hat{\beta}_{2}$} \\
\hline & & RB (\%) & EV & AVE & CP (\%) & RB (\%) & EV & AVE & CP $(\%)$ \\
\hline \multirow[t]{3}{*}{2} & 20 & -21.6 & 12.1 & 11.7 & 95 & -5.8 & 1.9 & 1.9 & 96 \\
\hline & 30 & -18.2 & 5.0 & 5.0 & 95 & -5.3 & 0.8 & 0.8 & 94 \\
\hline & 40 & -6.0 & 2.8 & 2.8 & 96 & -2.4 & 0.4 & 0.4 & 95 \\
\hline \multirow[t]{3}{*}{4} & 20 & -0.3 & 4.8 & 4.8 & 95 & -2.0 & 0.4 & 0.5 & 96 \\
\hline & 30 & -4.3 & 2.0 & 2.1 & 97 & -2.2 & 0.2 & 0.2 & 95 \\
\hline & 40 & -36 & 1.2 & 1.2 & 94 & -0.6 & 0.1 & 0.1 & 95 \\
\hline \multirow[t]{3}{*}{8} & 20 & 5.3 & 3.7 & 3.6 & 94 & -4.4 & 0.4 & 0.4 & 94 \\
\hline & 30 & 1.4 & 1.6 & 1.6 & 95 & -0.8 & 0.2 & 0.2 & 95 \\
\hline & 40 & -4.6 & 0.9 & 0.9 & 95 & -1.9 & 0.1 & 0.1 & 95 \\
\hline
\end{tabular}

NOTES: All entries in the EV and AVE columns are results after multiplication by $10^{3}$. 
Table 2

Maximum likelihood estimates of the regression coefficients in an analysis of the bark beetle data, along with standard errors and $p$-values, under four different models (a) periodic baseline hazards and time-homogeneous regression coefficients; (b) time-heterogeneous baseline hazards and time-homogeneous regression coefficients; (c) time-heterogeneous baseline hazards and time-heterogeneous regression coefficients; (d) time-heterogeneous baseline hazards and partially time-heterogeneous regression coefficients (homogeneous $\beta_{1 k}$ and heterogeneous $\beta_{2 k}$ ).

\begin{tabular}{|c|c|c|c|c|c|c|}
\hline & Estimate & Std. Error & $p$-value & Estimate & Std. Error & $p$-value \\
\hline & & Model (a) & & & Model (b) & \\
\hline$\beta_{1}$ & 0.50 & 0.05 & $<0.001$ & 0.52 & 0.05 & $<0.001$ \\
\hline$\beta_{2}$ & 0.32 & 0.01 & $<0.001$ & 0.35 & 0.01 & $<0.001$ \\
\hline \multirow[t]{2}{*}{ AIC } & & 2262.9 & & & 2181.5 & \\
\hline & & Model (c) & & & Model (d) & \\
\hline$\beta_{1}$ & - & & & 0.49 & 0.05 & $<0.001$ \\
\hline$\beta_{11}$ & 0.61 & 0.09 & $<0.001$ & - & & \\
\hline$\beta_{12}^{*}$ & -0.11 & 0.15 & 0.466 & - & & \\
\hline$\beta_{13}^{*}$ & -0.64 & 0.31 & 0.040 & - & & \\
\hline$\beta_{14}^{*}$ & -0.08 & 0.12 & 0.506 & - & & \\
\hline$\beta_{15}^{*}$ & -0.22 & 0.15 & 0.148 & - & & \\
\hline$\beta_{21}$ & 0.44 & 0.03 & $<0.001$ & 0.40 & 0.03 & $<0.001$ \\
\hline$\beta_{22}^{*}$ & -0.12 & 0.03 & $<0.001$ & -0.11 & 0.04 & 0.002 \\
\hline$\beta_{23}^{*}$ & -0.15 & 0.03 & $<0.001$ & 0.004 & 0.05 & 0.934 \\
\hline$\beta_{24}^{*}$ & 0.41 & 0.07 & $<0.001$ & 0.43 & 0.08 & $<0.001$ \\
\hline$\beta_{25}^{*}$ & -0.16 & 0.04 & $<0.001$ & -0.10 & 0.05 & 0.024 \\
\hline $\mathrm{AIC}$ & & 2158.5 & & & 2120.4 & \\
\hline
\end{tabular}

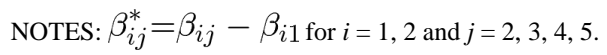

